\title{
ACS APPLIED BIO MATERIALS
}

Subscriber access provided by UNIV OF NEW ENGLAND ARMIDALE

\section{Article}

\section{Efficiency of antimicrobial electrospun thymol-loaded polycaprolactone mats in vivo}

Sara Garcia-Salinas, Enrique Gamez, Javier Asin, Ricardo de Miguel, Vanesa

Andreu, María Sancho-Albero, Gracia Mendoza, Silvia Irusta, and Manuel Arruebo

ACS Appl. Bio Mater., Just Accepted Manuscript • DOI: 10.1021/acsabm.0c00419 • Publication Date (Web): 23 Apr 2020

Downloaded from pubs.acs.org on April 26, 2020

\section{Just Accepted}

"Just Accepted" manuscripts have been peer-reviewed and accepted for publication. They are posted online prior to technical editing, formatting for publication and author proofing. The American Chemical Society provides "Just Accepted" as a service to the research community to expedite the dissemination of scientific material as soon as possible after acceptance. "Just Accepted" manuscripts appear in full in PDF format accompanied by an HTML abstract. "Just Accepted" manuscripts have been fully peer reviewed, but should not be considered the official version of record. They are citable by the Digital Object Identifier (DOI®). "Just Accepted" is an optional service offered to authors. Therefore, the "Just Accepted" Web site may not include all articles that will be published in the journal. After a manuscript is technically edited and formatted, it will be removed from the "Just Accepted" Web site and published as an ASAP article. Note that technical editing may introduce minor changes to the manuscript text and/or graphics which could affect content, and all legal disclaimers and ethical guidelines that apply to the journal pertain. ACS cannot be held responsible for errors or consequences arising from the use of information contained in these "Just Accepted" manuscripts. 


\title{
Efficiency of antimicrobial electrospun thymol-
}

\author{
loaded polycaprolactone mats in vivo
}

Sara García-Salinast,s, Enrique Gámezł, Javier Asín', Ricardo de Miguell, Vanesa

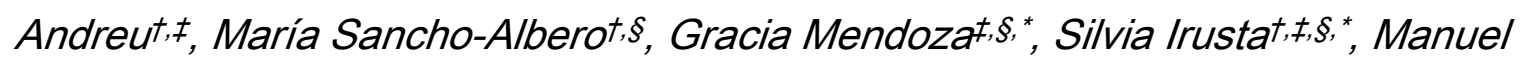

Arruebot, t, S

†Department of Chemical Engineering. Aragon Institute of Nanoscience (INA), University

of Zaragoza, Campus Río Ebro-Edificio I+D, C/ Poeta Mariano Esquillor S/N, 50018

Zaragoza, Spain

${ }^{\S}$ Networking Research Center on Bioengineering, Biomaterials and Nanomedicine,

CIBER-BBN, 28029 Madrid, Spain

"Department of Animal Pathology, Veterinary Faculty, University of Zaragoza, C/ Miguel

Servet, 177, 50013 Zaragoza, Spain

¥Aragon Health Research Institute (IIS Aragon), 50009 Zaragoza, Spain 
'Corresponding authors: Silvia Irusta (sirusta@unizar.es); Gracia Mendoza

(gmendoza@iisaragon.es) 
ABSTRACT. Due to the prevalence of antimicrobial resistant pathogens, natural products with long-term antimicrobial activity are considered as potential alternatives. In this work polycaprolactone $(P C L)$ electrospun fibers with mean diameters around $299 \mathrm{~nm}$ and loaded with $14.92 \pm 1.31 \% \mathrm{w} / \mathrm{w}$ of thymol (THY) were synthesized. The mats had appropriate elongation at break $(74.4 \pm 9.5 \%)$ and tensile strength $(3.0 \pm 0.5 \mathrm{MPa})$ to be potentially used as wound dressing materials. In vivo studies were performed using eight to ten-week-old male SKH1 hairless mice. The infection progression was evaluated through a semi-quantitative method and quantitative polymerase chain reaction ( $q P C R$ ). The analyses of post-mortem samples indicated that THY loaded PCL fibers acted as inhibitors of Staphylococcus aureus ATCC 25923 strain growth being as efficient as chlorhexidine (CLXD). Histopathological and immunohistochemical studies showed that the PCL-THY treated wounds were almost free of an inflammatory reaction. Therefore, wound dressings containing natural compounds can prevent infection, promote wound healing and prompt regeneration. 
KEYWORDS: wound dressing; electrospinning; PCL; thymol; chlorohexidine;

\section{Staphylococcus aureus}




\section{INTRODUCTION}

Injuries caused by burns, trauma or surgery are significant economic and social burden to healthcare providers. ${ }^{1}$ Wound dressings play an important role during the healing process and they have received growing attention in recent years..$^{2-4}$ In general, wound dressings are required to have good biocompatibility, provide a barrier against dust and bacteria, absorb exudates and debris and facilitate blood clogging while promoting transpiration avoiding wound maceration. ${ }^{5}$ It is also important for a wound dressing material to be as strong at least as human skin (tensile strength in the range 2-16 $\mathrm{MPa}$ ) to withstand mechanical stress to support the patient daily activities. ${ }^{5}$ Another expected characteristic is having adequate porosity to allow gas exchange but avoiding bacterial penetration acting as a physical barrier. ${ }^{1}$

Polymer nanofibers provide the possibility to immobilize antimicrobial compounds and their structure, similar to the extracellular matrix, has high interconnected porosity, and allows gas permeability. ${ }^{6}$ Among the different fibers fabrication techniques, electrospinning is the most commonly used method because of its versatility, cost- 
efficiency and straightforward setup. ${ }^{7}$ Synthetic (e.g., polycaprolactone (PCL), poly (Llactic acid) (PLLA), poly(lactic-co-glycolic acid), etc.) and natural polymers (e.g., polysaccharides, proteins, polyesters, etc.) have been used to produce electrospun nanofiber mats. Among the natural polymers, collagen nanofibrous matrices have been prepared and used in preclinical models demonstrating their superior improvement of the healing process. Microscopic examination revealed that early-stage healing in the group treated with these fibers was faster than that obtained for the control group. ${ }^{8}$ Also silk fibroin nanomatrices have demonstrated to accelerate re-epithelialization and wound closure in burns ${ }^{9}$ and collagen/chitosan composite membranes have promoted wound healing and induced cell migration and proliferation. ${ }^{10}$ In this sense, amoxicillin grafted onto regenerated bacterial cellulose sponges ${ }^{11}$ and hyaluronan/silver nanocomposites ${ }^{12}$ were also able to stimulate wound healing and reduce inflammation in different murine in vivo wound models.

However, the biodegradation rate and the relatively low mechanical strength displayed by natural polymers restrict their application as wound dressings despite of their reduced 
immune response and associated toxicity. ${ }^{13}$ For example, edible films developed from fruit and vegetable residue flour were reported to have a maximum tensile strength (TS) as low as $0.084 \mathrm{MPa} .{ }^{14}$ Polyvinyl acetate/chitosan/starch mats degradation in the first 7 days is reported to be in the range $15-30 \% .{ }^{15}$ Among the synthetic polymers, fibrous polyurethane membranes were evaluated as wound dressings and found to show appropriate oxygen permeability $\left(6.525 .10^{6}\right.$ Barrer $)$ while promoting fluid drainage. ${ }^{16}$ Silver/graphene composite hydrogels were also demonstrated to successfully enhance in vivo wound healing and tissue regeneration. ${ }^{17}$ Other synthetic polymers have been used to produce electrospun wound dressings, among them poly(lactic acid-co-glycolic acid) was found to produce mats with appropriate mechanical strength (tensile modulus from $39.23 \pm 8.15$ to $79.21 \pm 13.71 \mathrm{MPa}$ ) and porosity (38 to $60 \%$ ). ${ }^{18} \mathrm{PCL}$, a hydrophobic polyester polymer, has been widely used to prepare electrospun wound dressings because of its biodegradability, biocompatibility, chemical and thermal stability and mechanical properties. ${ }^{19}$ Furthermore, multicoated electrospun $\mathrm{PCL} /$ gelatin/nanosilver membranes have been recently shown as efficient antibacterial dressings in vivo by protecting wounds and promoting healing. ${ }^{20}$ Since bacterial infection is the most serious 
complication which might affect the wound healing process and can lead to impaired wound healing and increased morbidity and mortality, ${ }^{21}$ it is necessary to add antimicrobial agents to the wound dressing materials. However, it is also necessary to demonstrate that those advanced dressings are more effective than simple conventional dressings in clinical settings for the treatment of infected wounds. The electrospinning process allows the production in one step of drug loaded mats with the ability of providing a sustained release in the management of wound-associated infections.

The evolution of antimicrobial resistant pathogens that are refractory to the antibiotics of last resort represents a global public health challenge. ${ }^{22}$ Wound dressings containing natural products with long-term antimicrobial activity are considered as potential alternatives as cost-effective materials in combating antimicrobial resistance. ${ }^{23}$ In the last years, an increased number of publications on electrospun mats loaded with essential oils has been reported. ${ }^{24} \mathrm{~A}$ considerable number of these studies used pure bioactive compounds obtained from essential oils such as carvacrol and thymol (THY)..$^{25-29}$ We recently reported that carvacrol and thymol loaded electrospun polycaprolactone fibers are able to eliminate stationary phase concentration of Gram-positive (Staphylococcus 
aureus) and Gram-negative (Escherichia coll) bacteria in vitro. ${ }^{30}$ Therefore, as a continuation of that work we planned to carry out the in vivo evaluation of those advanced wound dressings.

Wounds in mice, infected with $S$. aureus, were also treated with poly(lactic-co-glycolic acid)/chitosan nanofiber wound dressings. ${ }^{31}$ Dressings containing hydroxypropyltrimethyl ammonium chloride were able to reduce the wound sizes by $21.8 \%$ after 3 days and by $100 \%$ after 15 days. Electrospun curcumin-loaded PCL-polyethylene glycol fibers have shown an efficiency on $\mathcal{S}$. aureus inhibition of $95 \%$ after $12 \mathrm{~h}$ treatment having also antiinflammatory effects in vitro. ${ }^{32}$ In vivo these mats improved wound healing by increasing fibroblast and vascular density and preventing oxidative damage.

In this manuscript, the mechanical properties of the thymol loaded PCL nanofibers were studied in order to confirm the potential applicability of the material as wound dressing. Then, the in vivo efficacy of the thymol loaded PCL nanostructured fibrous mats was tested in a full-thickness excision wound model in mice. The inhibition of bacterial growth 
in experimentally infected wounds was evaluated and histopathological examinations were conducted to investigate wound dressing effects.

\section{EXPERIMENTAL SECTION}

MATERIALS.

PCL $(M n=80,000 \mathrm{Da})$, dichloromethane $(\mathrm{DCM},>99 \%)$ and $\mathrm{N}, \mathrm{N}$-dimethylformamide

(DMF, > $99 \%$ ) were purchased from Fisher Scientific. Thymol (99\%) was purchased

from Acros Organics while phosphate-buffered saline (PBS), (S)-(-)-limonene (food grade, $\geq 95 \%)$, naproxen sodium salt (98-102\%), and Tween 80 were obtained from Sigma-Aldrich. Acetonitrile ( $\geq 99.9 \%$ ), formic acid (98-100 \%), and deuterated chloroform $(99.8 \%$ D) were acquired from VWR. All reagents were used as received without any further purification. Chlorhexidine Gluconate $1 \%$ was purchased from Salvat.

METHODS

PCL and THY loaded PCL fibers preparation. 
The preparation of fiber mats was carried out as previously described. ${ }^{33}$ Briefly, PCL was dissolved in a mixture of DCM and DMF (at 1:1 volume ratio), for the THY loaded fibers, the appropriate amount of THY was added to the polymer solution ( $20 \mathrm{w} / \mathrm{w} \%$ referred to the PCL mass). The electrospinning process was carried out in an Yflow 2.2 D500 electrospinner equipped with a flat collector covered with aluminum foil. The solution was fed with a flow rate of $1.0 \mathrm{~mL} / \mathrm{h}$. The tip to collector distance was $18 \mathrm{~cm}$. The voltage applied to the collector was fixed at $-4 \mathrm{kV}$ while the voltage applied to the needle was 10.25 kV for PCL and $12.13 \mathrm{kV}$ for the THY loaded fibers, respectively.

\section{Physico-chemical and mechanical characterization of prepared materials}

The morphology of the electrospun mats was analysed in a CSEM-FEG INSPECT 50, FEI scanning electron microscope (SEM). Fibers mean diameter was determined measuring at least 100 nanofibers from 3 independent SEM images. Samples were previously covered with an Au/Pd layer to allow electronic observation. THY loading was determined in a Shimadzu 2010SE GC-MS chromatograph equipped with an AOC 20i injector. Samples were previously dissolved in a mixture of DCM and acetonitrile and an 
internal standard ((S)-(-)-limonene) was added. The encapsulation efficiency (EE) was

calculated with the following equation:

$E E=\frac{T H Y \text { measured amount }}{T H Y \text { theoretical amount }} x 100$

[Eq 1]

The theoretical amount was calculated based on the THY/PCL ratio used in the electrospinning solution. THY release tests were carried out in a continuous mode by flushing a solution of $2 \% \mathrm{w} / \mathrm{v}$ of Tween 80 in PBS at $37 \stackrel{\circ}{\circ} \mathrm{C}$ through the samples with a flow rate of $1 \mathrm{~mL} / \mathrm{min}$ using a Shimadzu LC-10AT VP syringe pump. Released samples were collected and analyzed using an Acquity UPLC $®$ Waters liquid chromatograph with a photodiode array detector ACQ-PDA. Naproxen sodium salt was used as internal standard.

Mechanical properties of the mats were tested using an Instron Microtester 5548 and a video extensometer laser without contact (Instron 2663-281). Stress-strain curves were recorded at a stretching speed of $1 \mathrm{~mm} / \mathrm{min}$. The dimensions of the tested probes were in agreement with the ISO 527-1:2012 norm (Plastics - Determination of tensile properties). 
Mercury porosimetry was used to evaluate the pore volume of the electrospun mats. A mercury porosimeter MicroActive AutoPore V9600 from Micromeritics Instrument Corporation. Dried and degassed $2.5 \mathrm{~cm}$ long squared $\mathrm{PCL}$ electrospun samples were used in the evaluation.

Mouse excisional wound splinting model and infection

In vivo studies were performed under Project License 51/14 approved by the Ethic Committee for Animal Experiments of the University of Zaragoza (Spain). In these studies, eight to ten-week-old male SKH1 hairless mice (Charles River Laboratories) were used. Mice were fed ad libitum and maintained under specific pathogen-free conditions accordingly with the Spanish Policy for Animal Protection RD53/2013, which meets the European Union Directive $2010 / 63$ on the protection of animals destined to scientific purposes.

Thirty mice were experimentally divided in five groups $(\mathrm{N}=6)$ : i) Control group: Wounds without infection or treatment; ii) PCL group: Wounds infected and treated with $\mathrm{PCL}$ dressings; iii) PCL-THY group: Wounds infected and treated with thymol-loaded PCL 
dressings; iv) THY group: Wounds infected and treated with free THY; v) CLXD group:

Wounds infected and treated with chlorhexidine. In each group, three mice were euthanized at 3 days post-surgery and infection (dpi) and other three mice at $7 \mathrm{dpi}$.

The mouse excisional wound splinting model ${ }^{34}$ with some modifications was developed to evaluate wound infection and healing while avoiding the natural murine wound closure through skin contraction with the purpose to mimic the granulation and reepithelization processes that take place during human wound healing 35 (Fig. 1).

In order to evaluate potential weight loss during the experiments, the animals were daily weighted. For the surgical procedure, SKHI hairless mice were initially anesthetized with $5 \%$ isoflurane, and maintained with $1-2 \%$ isoflurane ( $1 \mathrm{~L} / \mathrm{min}$ oxygen flow). The mice were rinsed with a $70 \%$ ethanol $(\mathrm{v} / \mathrm{v})$ swab to be sterilely prepped. Meloxicam $(2.5 \mathrm{mg} / \mathrm{kg}$ body weight) was then subcutaneously administered for pain relief (daily until $48 \mathrm{~h}$ postsurgery). A sterile 8-mm punch biopsy tool (E ickemeyer Veterinary Equipment Ltd.) was employed to pattern two full-thickness wounds in the skin of the dorsum at each side of the median line of the animal. After that, two donut-shaped silicone wound splints (Grace 
Bio-Labs) were sutured with six interrupted $4 / 0$ sutures (Braun) to avoid the natural murine wound closure through skin contraction. Wounds were subsequently infected by inoculation of $10^{7}$ colony forming units (CFU; $25 \mu \mathrm{L}$ in PBS) of $S$. aureus ATCC 25923 (lelab). Different treatments were then applied: PCL dressing mats (as control of infection), THY loaded PCL dressing mats, free THY or free chlorhexidine (CLXD; as model antiseptic in clinical use). The dressing mats diameter was $12 \mathrm{~mm}$ whereas free compounds were added in a volume of $25 \mu \mathrm{L}$, which corresponds to the amount of THY loaded in $12 \mathrm{~mm}$ of PCL-THY dressings and CLXD was added at the concentration used in the current clinical practice $(10 \mathrm{mg} / \mathrm{mL})$. Free THY was also assessed to compare the effect of THY in wound infection and healing when encapsulated in the PCL-based mat vs the free compound. Finally, wounds and dressings were covered with sterile adhesive plasters and bandages (Hartmann). Dressing mats were replaced every day for 3 days, then all wounds were uncovered, as recommended in the clinical practice. ${ }^{36-37}$ The progression of infection as well as weight loss and potential pain were monitored daily until the end of the studies. 


\section{Evaluation of infection in wounds}

The infection progression in wounds was evaluated through a semi-quantitative analysis of microbiological cultures and quantitative polymerase chain reaction ( $q P C R$ ). The microbiological results were obtained from three independent experiments run in triplicate.

Microbiological samples were harvested from wounds by means of microbiological swabs with Amies media (Deltalab) at 1, 2, 3 and $7 \mathrm{dpi}$. The microbiological samples were cultured on blood agar and McConkey No. 3 media (Oxoid). After incubation ( $37^{\circ} \mathrm{C}, 24$ h), bacteria concentration in the samples was semiquantified and the microorganism identified by reseeding samples and analyzing by a MALDI-TOF system (Bruker). Concurrently, qPCR evaluation of $S$. aureus ATCC 25923 was carried out in the samples. Briefly, DNA was obtained (DNeasy Blood \& Tissue Kit, Qiagen) and amplified through the EXO one Staphylococcus aureus one MIX qPCR kit (Exopol) and a 7500 FAST Real Time PCR System (Applied Biosystems). The pre-incubation step (1 cycle, 5 min, $95{ }^{\circ} \mathrm{C}$ ) 
was followed by an amplification stage of 42 cycles of $15 \mathrm{~s}$ at $95^{\circ} \mathrm{C}$ and $1 \mathrm{~min}$ at $60^{\circ} \mathrm{C}$, to read then the plate. 
(1)

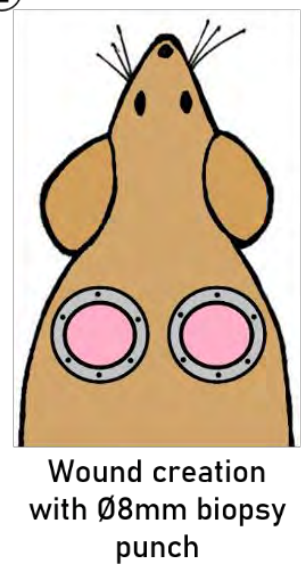

(2)

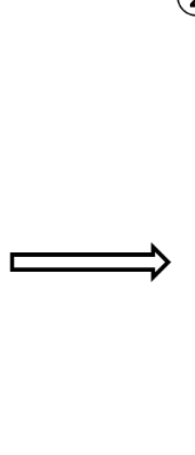

$$
\text { wo }
$$

CFU of
Staphylococcus aureus
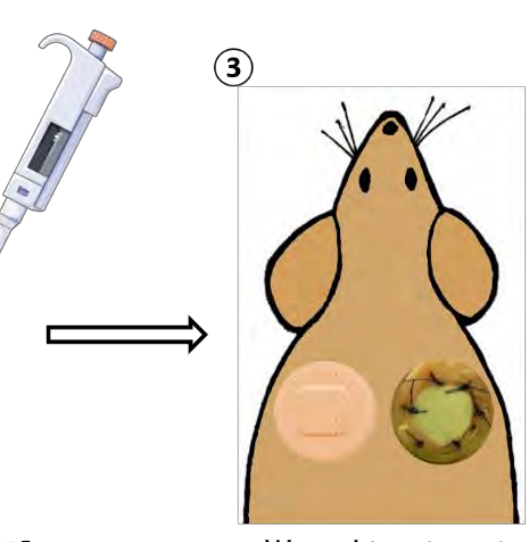

Wound treatment

Figure 1 Schematic representation of the in vivo wound model: 1) Splinting wounds were surgically performed with an 8-mm-diameter biopsy punch and the splinting ring sutured around the wound; 2) Induction of the infection was achieved by the inoculation of Staphylococcus aureus ( $10^{7}$ colony forming units (CFU)); 3) Wound treatment was carried out by adding the different synthesized mats and covering the wounds.

\section{Histopathologic studies}

Euthanasia was carried out by $\mathrm{CO}_{2}$ inhalation after 3 and $7 \mathrm{dpi}$. Then, wounds were totally exposed by removing splints, sutures, dressings and gauzes, and harvested together with $\sim 5 \mathrm{~mm}$ in diameter of surrounding tissue. Samples were then fixed for $24 \mathrm{~h}$ in paraformaldehyde (4\%; Alfa Aesar) and embedded in paraffin. Five $\mu \mathrm{m}$ sections were 
stained with hematoxylin and eosin (HE), and Gram staining for histopathological and bacteria determination, respectively. In order to assess wound angiogenesis, an immunohistochemical evaluation was performed by using rabbit polyclonal CD31 antibody (ab28364, Abcam). The automated immunostaining platform Autostainer Link (Dako) was used. The slides were dewaxed in xylene and re-hydrated in an ethanol series. Antigen retrieval was carried out by high pH buffer treatment (CC $1 \mathrm{~m}, \mathrm{R}$ oche) and $3 \% \mathrm{H}_{2} \mathrm{O}_{2}$ was added to block the endogenous peroxidase. Subsequently, the slides were incubated with the primary antibody (1:50 for $60 \mathrm{~min}$ ) followed by the corresponding visualization system conjugated with horseradish peroxidase (EnVision FLEX+, Dako). The chromogen 3, 30-diaminobenzidine tetrahydrochloride (DAB) was used for the detection of the immunohistochemical reaction. Nuclei staining were carried out using Carazzi's hematoxylin. Finally, the slides were dehydrated and permanent mounted.

\section{Statistical analysis}

All data are reported as mean $\pm S D$. The significant differences among the means were analyzed by the two-way analysis of variance (ANOVA) for multiple comparisons by 


\author{
Dunnett's multiple comparisons test (GraphPad Software). Statistically significant \\ differences were considered when $p \leq 0.05$.
}

\title{
RESULTS AND DISCUSSION
}

PHYSICOCHEMICAL AND MECHANICAL CHARACTERIZATION. The morphology of

PCL and THY loaded PCL nanofibers was characterized by SEM (Fig. 2). The mean

diameter of unloaded fibers ( $266 \pm 73 \mathrm{~nm}$ ) show no significant changes when THY was

incorporated to the spinning solution $(299 \pm 71 \mathrm{~nm})$. Similar results were previously found

in our preceding work also for carvacrol loaded PCL fibers. ${ }^{33}$ 

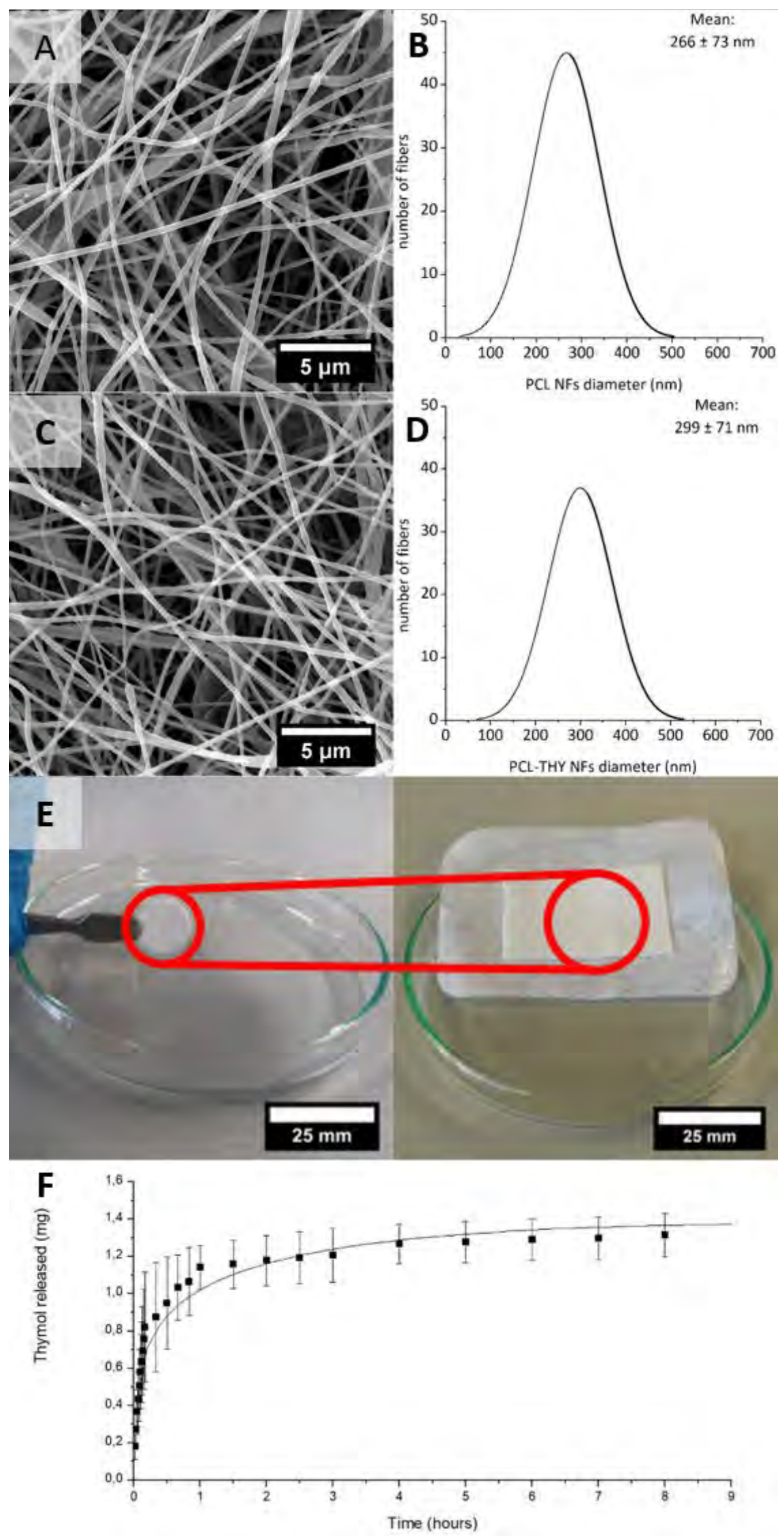
Figure 2 Characterization of electrospun nanofibers: A, B) SEM micrograph and diameter histogram of PCL nanofibers (Number of fibers measured=100); C, D) SEM micrograph and diameter histogram of THY loaded PCL nanofibers; E) Macroscopic visualization of the synthesized THY loaded PCL nanofibers. The diameter of the selected section of the dressing is $1 \mathrm{~cm}$. F) Thymol release profile from THY loaded PCL nanofibers. Mean \pm SD; 18 data per time point.

Mercury porosimetry revealed a pore size in the randomly oriented PCL nanofibers considering the $\mathrm{Hg}$ intrusion of $1.9 \mu \mathrm{m}$ and a total porosity (pore volume/total volume) of $73 \%$. THY incorporation in the fibers did not influence the structural analysis of the fibers.

Electrospun nanofibers can act as drug delivery systems that exhibit sustained drug release profiles, leading to a potential reduction in the frequency of the treatments when topical applications are envisaged. Besides, it is known that the electrospinning technique allows high drug loading capacities due to the large area per volume ratio and high specific surface area of nanofibrous materials. ${ }^{33}$ In addition, during the electrospinning process in the flight from the needle to the collector the organic solvent is rapidly 
evaporated with a consequent minimal loss of the dissolved drug. A THY load of $14.92 \pm$

$1.31 \% \mathrm{w} / \mathrm{w}$ was achieved by incorporation of the essential oil compound to the electrospinning solution, which is in the range of previous works $(0.4-35 \%)$ though some variability in THY load is reported among them. ${ }^{38-39}$ This value indicates an encapsulation efficiency of $74.62 \%$, similar to the encapulation efficiency (EE) previously reported for essential oil (EO) compounds encapsulated in PCL electrospun fibers. ${ }^{30,40-41}$

The in vitro release of THY from the PCL fibers is shown in Fig. $2 F$. The initial burst release observed in the first minutes would be related to the EO compound present on the external surface of the fibers. ${ }^{42}$ This initial release was followed by a controlled release over $8 \mathrm{~h}$ and only $8.81 \%$ of the loaded THY was released during this time in agreement with the PCL ability to provide with a sustained release. ${ }^{43}$ Drug release from nanofibers could be caused by its desorption from the fibers surface, diffusion from the pores or matrix degradation. ${ }^{44}$ In this case the burst release would be attributed to the desorption from the surface while the sustained release observed would be due to diffusion from the 


\begin{abstract}
PCL matrix through pores since no fibers degradation was observed after one week in
PBS (data not shown).
\end{abstract}

The THY release data were treated with Weibull, Korsmeyer-Peppas, Peppas-Sahlin, and

Ritger-Peppas kinetic models (Table SI). Peppas and Sahlin model was found to be the best

fit, as it presents the highest $\mathrm{R}^{2}$ correlation coefficient $(0.945)$. In this model one term $\left(\mathrm{k}_{1} . \mathrm{t}^{\mathrm{n}}\right)$

represents the Fickian diffusional contribution which occurs by the usual molecular

diffusion of the drug due to a chemical potential gradient. The second term $\left(k_{2} \cdot t^{2 n}\right)$

represents the case-II relaxation contribution associated with polymer chains relaxation. ${ }^{45}$

The constant values found $\left(\mathrm{k}_{1}=2.63 \mathrm{~s}^{-\mathrm{n}}\right.$ and $\left.\mathrm{k}_{2}=-0.184 \mathrm{~s}^{-2 \mathrm{n}}\right)$ clearly indicate the predominance of the Fickian diffusion in the release process. It is also corroborated by the negative value obtained for $\mathrm{k}_{2}$. In accordance to Peppas-Sahlin equation the value of the exponent $n$ for a Fickian release mechanism from polymeric systems having cylindrical geometries should be around 0.43 . Values lower than 0.45 would be related to the wide fibers' diameter dispersion.

Since the wound dressing materials being wrapped on the wound area are likely to be subjected to pulling forces in order to adhere the mat smoothly and effectively to the skin, they are expected to have similar mechanical strength and elasticity than normal human 
skin. Tensile strength of human skin is in the range 2-16 MPa and its elongation-at-break in the $70-77 \%$ range. ${ }^{46}$ One of the significant features of PCL for biomedical application is its high elongation-at-break, ${ }^{47}$ in our case, the measured value of $108.6 \pm 11.3 \%$ is much higher than the one of the human skin. The tensile strength retrieved of $5.1 \pm 0.5$ $\mathrm{MPa}$ would be also in the required range for wound dressing applications. This value decreased to $3.0 \pm 0.5 \mathrm{MPa}$ with the addition of THY, but it is still in the appropriate applicability range. Similar results were obtained from the elongation-at-break of THY loaded PCL fibers, the value was reduced to $74.4 \pm 9.5 \%$. It is known that the mechanical properties of pure polymers can be varied by incorporating bioactive compounds. It has been reported that increasing the concentration of cinnamon in $\mathrm{PCL} /$ gelatin fibers decreased the tensile strength as a result of an improved porosity. ${ }^{48}$ But this is not the case of our mats, since, as mentioned before, THY addition did not influence the structural analysis of the fibers. PCL molecular chains are likely to be more uneven and disordered due to the presence of THY, resulting in reduced mechanical properties. ${ }^{49}$ However, the mechanical properties of the THY loaded PCL mats here reported are mechanically appropriate for wound dressing applications. 
IN VIVO BACTERICIDAL CAPACITY. Both, acute and chronic wound infections, are severe complications worldwide that delay and complicate wound healing. If the host defense is no capable of overcoming the bacterial burden, an infection takes place, causing delayed wound healing, inflammation and tissue damage. ${ }^{50}$ Since extensive abuse of antibiotics in wound care has led to new pathogens occurrence and the prevalence of multi-resistant bacteria, the use of natural components as antimicrobial and antiseptic agents is steadily growing. Herein we evaluated the bactericidal efficiency of the prepared mats (PCL-THY group) in an in vivo model of infected wounds and compared to unloaded fibers (PCL group), free thymol (THY group) and free chlorhexidine (CLXD group) as disinfectant and antiseptic model widely used on the skin. Twelve mmdiameter disks, with the necessary weight to reach the minimum bactericidal concentration $(\mathrm{MBC})$ value found previously by our group in in vitro assays on Staphylococcus aureus ATCC 25923 ( $30 \mathrm{mg}$ of dressing containing $4.48 \mathrm{mg}$ of THY) were evaluated. ${ }^{33}$ 
None of the animals presented changes in behavior or showed any signs of physical discomfort, but a purulent secretion was observed in the PCL group as can be seen in the visual evolution photographically recorded at 7 dpi (days post-infection; Fig. 3A). Microbiological swab analysis results are shown as insets in Fig. 3A. Animals treated with unloaded fibers (PCL group) presented massive bacterial growth at any time analysed. After one day post-surgery and infection, the application of a single dose of CLXD seemed to be the most effective treatment since no growth was observed from the collected swabs while mild bacterial growth were detected in wounds treated with THY loaded mats (PCLTHY group). On the other hand, free THY was less effective and a moderate bacterial growth was observed. After 2 days, results were similar except for the CLXD group that showed the presence of a mild bacterial growth. In the third day, a decrease in the media of colonies counted was observed for the THY group. After this time, no treatment was applied to the wounds and, as a consequence, massive bacterial growth was observed in the THY group. PCL-THY and CLXD groups showed a moderate bacterial growth; however, no massive growth was found. These high loadings, even observed for the 
commonly used commercially available CLXD used at the clinical concentration available, may be attributed to the enormous bacterial challenge that was used ( $\left.4 \times 10^{8} \mathrm{CFU} / \mathrm{mL}\right)$. 

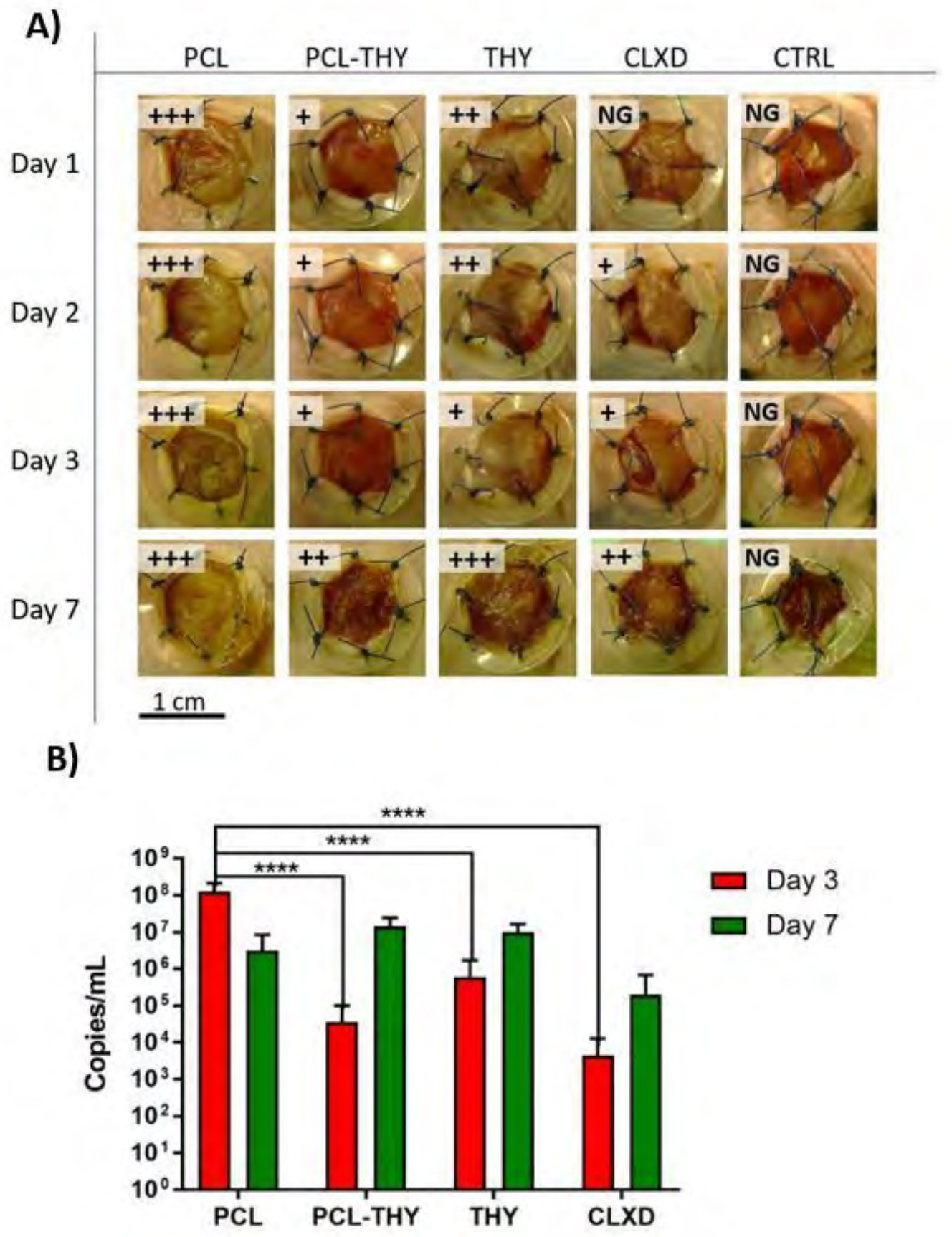

Figure 3 In vivo wound infection model in SKH1 mice and treatment with the electrospun mats (PCL and PCL-THY groups), free THY and the model antiseptic CLXD: A) Wounds evolution at 1, 2, 3 and $7 \mathrm{dpi}$ with $\mathcal{S}$. aureus ATCC 25923. Microbiological results in 
experimental and control groups are showed as insets. NG No growth; (+) Mild bacterial growth; (++) Moderate bacterial growth; (+++) Massive bacterial growth. Scale bar is the same for all wounds in the figure. B) Microbiological $\mathrm{QPCR}$ results in experimental and control groups. Statistics compares PCL-THY, THY and CLXD groups with PCL group. **** refers to $p$ value $<0.0001$

Quantitative PCR analyses of post-mortem skin samples (Fig. 3B) indicated that, after three days of treatment, PCL-THY and CLXD groups showed at least two log-reduction in the number of $S$. aureus ATCC 25923 strain copies. These results would indicate that THY loaded PCL fibers act as inhibitors of this bacterial strain growth being as efficient as the model antiseptic used CLXD. However, our previous studies showed the in vitro detrimental effects of CLXD treatment in different human cell cultures, ${ }^{51}$ resulting in cell viability percentages lower than $70 \%$ at the lowest concentration tested $(4 \mu \mathrm{g} / \mathrm{mL})$ which were dramatically decreased to $20 \%$ from $15 \mu \mathrm{g} / \mathrm{mL}$. On the other hand, THY treatment did not show cytotoxic effects (viability $\geq 70 \%$ ) in these cell lines up to concentrations 
higher than $60 \mu \mathrm{g} / \mathrm{mL}$. These results point to the more cytocompatible nature of THY compared to CLXD.

A large amount of reports and experimental evidence sustain the beneficial properties of essential oil compounds on promoting wound healing. ${ }^{52}$ For example, electrospun mats based on PCL, PLA, and 50/50 hybrid composites were loaded with THY and their effects evaluated in an in vivo rat wound model by Karami et al. ${ }^{53}$ Their findings pointed to a significant better performance of the PLA/PCL hybrid membranes loading THY at the end of the experiments (14 days of treatment) regarding granulation tissue formation and reepithelialization compared to commercial dressings and gauze bandages. In this sense, THY enriched collagen hydrogels ${ }^{54}$ and bacterial cellulose hydrogels ${ }^{55}$ were previously reported as novel and efficient composite dressing mats in the in vivo healing of different rat models. Collagen-based films loading THY (1.2 mg of THY per dressing) clearly reduced inflammation and enhanced regeneration in surgical 8-mm wounds in a rat model after 7 days of treatment, highlighting the presence of mature granulation tissue due to the presence of well-formed and dilated vessels. ${ }^{54}$ On the other hand, the in vivo effects 
of THY loaded bacterial cellulose hydrogels as dressings in a burn wound model also showed a decreased inflammatory reaction in the groups treated with the hydrogels loading THY compared to control groups after treatment for 15 days. ${ }^{55}$ Both studies pointed to the potential stimulation of skin regeneration through the formation of granulation tissue due to the proliferative effects of THY in fibroblast and enhanced collagen deposition. Additionally, some studies confirm the benefits of nanostructured materials compared with commercially available wound dressings. For example, the antimicrobial peptide Tet213 immobilized onto a substrate of alginate, hyaluronic acid and collagen nanosctructured composite presents a better wound closure rate when compared with commercial Aquacel Ag wound dressing after 7 days since wound infection. Also, bacterial presence in wound was lower when treated with this composite material when compared with Aquacel Ag-treated wounds after 3 days. ${ }^{56}$ Another study shows the potential use of electrospun nanofibers based on honey, polyvinyl alcohol and chitosan, enriched with the aqueous extracts of Cleome droserifolia and Allium sativum as antimicrobial wound dressings. Results show a superior in vitro antibacterial activity against $S$. aureus of the synthetized nanofibers compared with commercial Aquacel Ag. 
This study also shows a faster wound closure when using the synthetized nanofibers compared to the timing needed to reach closure with Aquacel Ag treated on infected wounds. 57

Histopathological and immunohistochemical studies of treated wounds were carried out to evaluate the effects of our mats related to infection, angiogenesis, and tissue regeneration (Fig. 4-6). The most important lesions were observed for the PCL group (Fig. 4A) and consisted on severe diffuse necrotizing dermatitis in the wound area that was characterized by massive infiltrations of inflammatory cells (lymphocytes and macrophages) together with severe tissue necrosis. Inflammatory reaction affected all layers of the skin, reaching the adipose tissue (panniculitis). Throughout the skin layers but mostly on the surface, abundant colonies of coccoid bacteria were observed ( $F$ ig. 4A). The PCL-THY group showed wounds that were almost free of inflammation reaction, with only a few layers of coagulative necrosis on the surface of the exposed area of the wounds (Fig. 4B). The THY group showed a less intense, multifocal inflammatory reaction when compared to the inflammation caused in the PCL group (Fig. $4 \mathrm{C}$ ). 

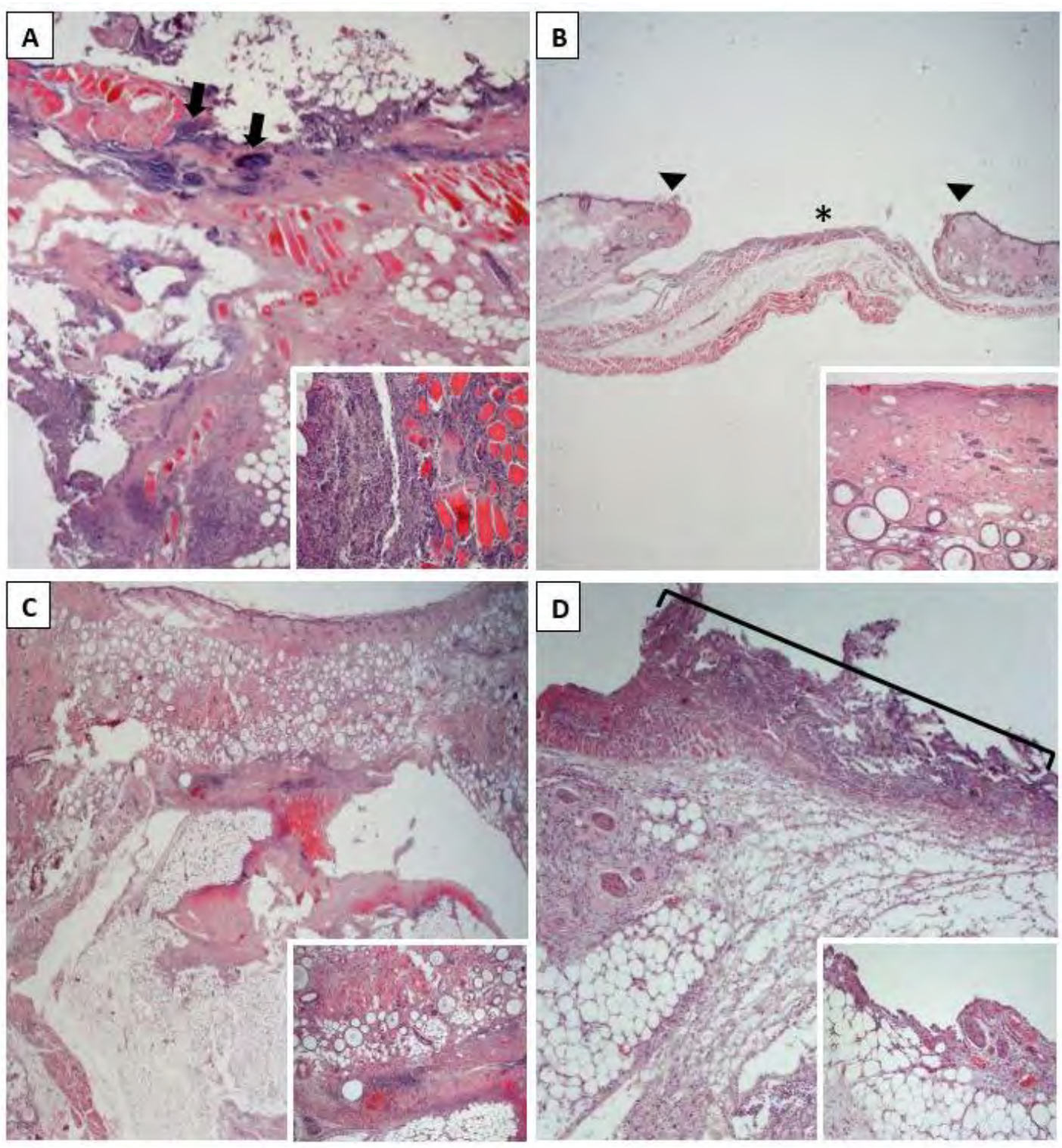

Figure 4 Histological analysis of skin wounds, representative images at 3 dpi (days postinfection). A) PCL group. Severe diffuse necrotizing dermatitis and panniculitis. Clusters of coccoid bacteria are observed in the superficial layers (arrows). Inset: Detail of the inflammatory reaction, with numerous lymphocytes and macrophages around muscle 
fibers; B) PCL-THY group. Section of the wound and wound edges (arrowhead). No inflammatory reaction is observed in the exposed dermis (asterisk) or in deep layers of the skin. Inset: Absence of inflammation in another area of the dermis in the same animal; C) THY group. Focal, less severe inflammatory reaction in the panniculus. Inset: Detail of the deep inflammatory reaction; D) CLXD group. Absence of inflammation in the dermis. Exposed dermal surface presents an important superficial layer of coagulative necrosis (square bracket). Inset: Detail of another area of the same animal, showing lack of inflammation. Hematoxylin-eosin staining, $1 x$, insets at 20x.

Finally, the CLXD group also showed absence of inflammatory reaction but a much thicker layer of coagulative necrosis on the exposed surface of the wound (Fig. 4D), which agrees with the in vitro detrimental findings previously reported by our group. ${ }^{51}$ Coccoid bacteria were only observed in sections of the PCL and THY groups (Fig. 5). Finally, the semi-quantitative analysis of angiogenesis performed with rabbit polyclonal CD31 antibody showed a homogeneous increase in the number of blood vessels at $7 \mathrm{dpi}$, that was similar for all infected groups (Fig. 6). 


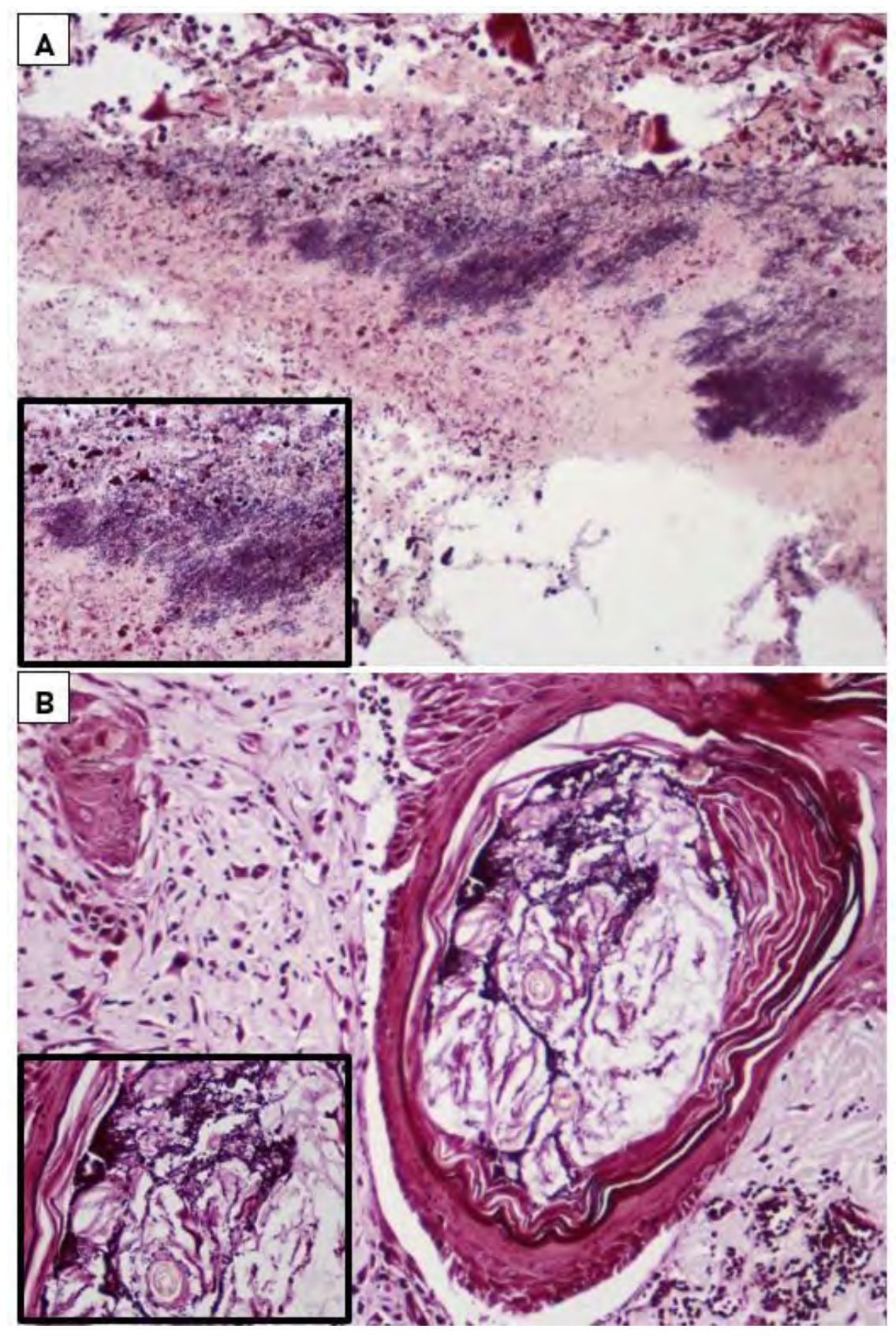

Figure 5 Detection of bacteria in skin wounds. Representative images of PCL and THY groups after 3 days of surgery and infection. PCL-THY and CLXD groups are not 
represented due to the lack of bacteria. (A) PCL group. Massive growth of coccoid bacteria in clusters in superficial layers of the skin together with severe tissue necrosis. Inset: Detail of the bacteria in the same animal. (B) THY group. G rowth of coccoid bacteria within a hair follicle. Inset: Detail of the bacteria. Gram staining, 20x, insets at 60x. 


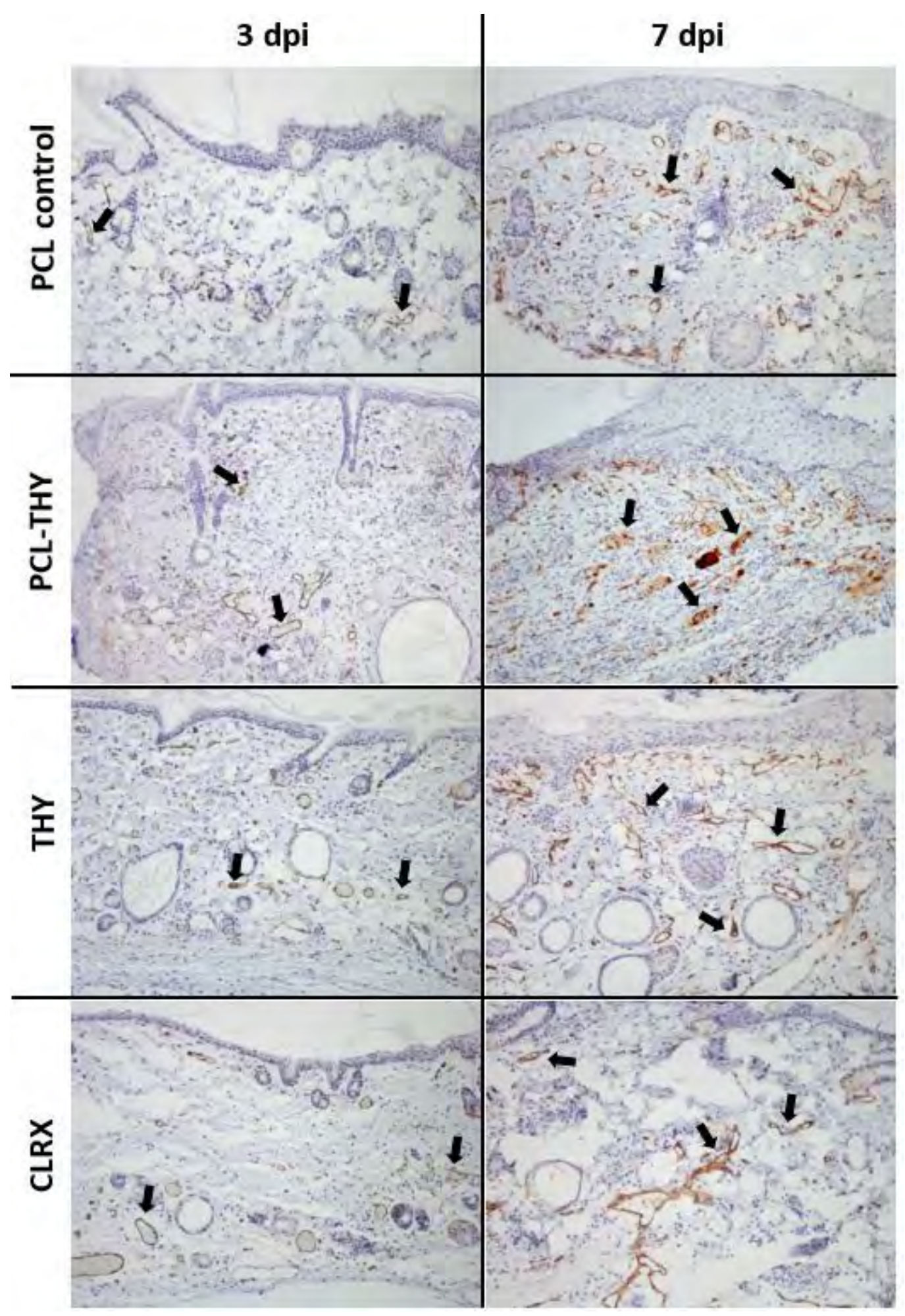


Figure 6 Location of blood vessels in skin wound samples, representative images at 3 and $7 \mathrm{dpi}$. All groups showed an increased number of blood vessels at $7 \mathrm{dpi}$ compared with samples at $3 \mathrm{dpi}$. Immunohistochemistry for CD 31, 20x.

Our results highlight the benefit of using THY-releasing mats over the use of just chlorhexidine, not only to reduce the high cytotoxic effect of the later but also because the mats provide with a slow release of the incorporated THY. Usually wound dressings are frequently replaced and the potential local toxicity of repeated exposure to chlorhexidine could be avoided by the use of the THY-loaded mats here reported. Chlorhexidine at the clinical recommended concentration produces a successful elimination of microbial contamination on the wound bed, but its action is not prolonged over time and repeated administrations might delay wound healing. Conversely, the THYloaded wound dressings here reported can release and extend their antimicrobial action until the subsequent dressing replacement.

CONCLUSIONS 
Mats consisting on fibers with homogenous diameters distribution (299 $\pm 71 \mathrm{~nm})$ and high THY loading $(14.92 \pm 1.31 \% \mathrm{w} / \mathrm{w})$ were obtained by electrospinning with high encapsulation efficiencies. Tensile strength and elongation at break of the prepared materials were tested and the values found were $3.0 \pm 0.5 \mathrm{MPa}$ and $74.4 \pm 9.5 \%$ respectively, which make them appropriate for wound dressing applications. In vivo tests to evaluate the antimicrobial action of the mats showed that animals treated with unloaded fibers presented massive growth at any time analysed. On the contrary, one day postsurgery and infection, few colonies were detected in wounds treated with THY loaded mats while a high number of colonies appeared in wounds treated with free THY, showing the importance of drug encapsulation and the need of contact between bacteria and the mat to generate a superior antimicrobial action. After treatment discontinuation, massive bacterial growth was observed in the THY group while for PCL-THY and CLXD treated wound no massive growths were found. Histopathological and immunohistochemical studies of wounds showed severe diffuse necrotizing dermatitis in the wound area that was characterized by massive infiltrations of inflammatory cells and severe tissue necrosis in the PCL treated wounds. In addition, massive growths of coccoid bacteria 


\begin{abstract}
were observed in these tissues. The PCL-THY treated wounds were almost free of inflammatory reaction, with only a few layers of coagulative necrosis on the surface of the exposed area of the wounds. In comparison, CLXD treated wounds showed a much thicker layer of coagulative necrosis in the exposed surface of the wound. These results show that PCL-THY mats are able to control bacterial infection as efficiently as the model antiseptic CLXD though significantly diminishing tissue damage, highlighting their potential biomedical application.
\end{abstract}

\title{
Supporting Information
}

Figure SI: Examples of stress-strain curves for A) PCL nanofibers, B) THY loaded PCL nanofibers

Table SI: Kinetic release models and parameters obtained

\author{
AUTHOR INFORMATION
}

Corresponding Author

* Silvia Irusta (sirusta@ unizar.es); Gracia Mendoza (gmendoza@iisaragon.es) 


\author{
Author Contributions \\ The manuscript was written through contributions of all authors. All authors have given \\ approval to the final version of the manuscript.
}

\title{
Present Addresses
}

J.A. present address: California Animal Health and Food Safety Laboratory System (CAHFS), University of California, Davis, San Bernardino, CA

\section{Funding Sources}

Spanish Ministry of Economy and Competitiveness (grant number CTQ2014-52384-R).

EU (ERC Consolidator Grant program, ERC-2013-CoG-614715). Instituto de Salud Carlos III (Spain).

\section{ACKNOWLEDGMENT}


This work was funded by the Spanish Ministry of Economy and Competitiveness (grant number CTQ2014-52384-R). We also acknowledge financial support from the EU (ERC Consolidator Grant program, ERC-2013-CoG-614715, NANOHEDONISM). CIBER-BBN is financed by the Instituto de Salud Carlos III (Spain) with assistance from the European Regional Development Fund. We are grateful to LMA-INA (University of Zaragoza, Spain), the Histopathology Unit from CNIO (Madrid, Spain), and Cell Culture, Animal Care and Pathological Anatomy Core Units from IACS/IIS Aragon (S pain) for their instruments and expertise. We gratefully acknowledge Dr Elena Tapia, Dr Montserrat Perez-Piñero, Dr Ander Izeta, and Dr Laura Yndriago for helpful advice and comments regarding the in vivo wound model. S.G-S. also acknowledges the support from the FPI program (BES2015-073735). M.S-A thanks the Spanish government for the FPU PhD research fellowship. G.M. gratefully acknowledges the support from the Miguel Servet Program (MS 19/00092; Instituto de Salud Carlos III).

\section{REFERENCES}


1. Li, S.; Li, L.; Guo, C.; Q in, H.; Yu, X., A promising wound dressing material with excellent cytocompatibility and proangiogenesis action for wound healing: Strontium loaded Silk fibroin/S odium alginate (SF/SA) blend films. International Journal of Biological Macromolecules 2017, 104, 969-978.

2. Kamoun, E. A.; Kenawy, E.-R. S.; Chen, X., A review on polymeric hydrogel membranes for wound dressing applications: PVA-based hydrogel dressings. Journal of Advanced Research 2017, 8 (3), 217-233.

3. Curvello, R.; Raghuwanshi, V. S.; Garnier, G., Engineering nanocellulose hydrogels for biomedical applications. Advances in Colloid and Interface Science 2019, $267,47-61$.

4. Miguel, S. P.; Moreira, A. F.; Correia, I. J ., Chitosan based-asymmetric membranes for wound healing: A review. International Journal of Biological Macromolecules 2019, 127, 460-475. 
5. Feng, Y.; Li, X.; Zhang, Q.; Yan, S.; Guo, Y.; Li, M.; You, R., Mechanically robust and flexible silk protein/polysaccharide composite sponges for wound dressing. Carbohydrate Polymers 2019, 216, 17-24.

6. Rasouli, R.; Barhoum, A.; Bechelany, M.; Dufresne, A., Nanofibers for Biomedical and Healthcare Applications. Macromolecular Bioscience 2019, 19 (2).

7. Liao, S.; Li, B. J .; Ma, Z. W.; Wei, H.; Chan, C.; Ramakrishna, S., Biomimetic electrospun nanofibers for tissue regeneration. Biomedical Materials 2006, 1 (3), R 45-

R53.

8. Rho, K. S.; J eong, L.; Lee, G.; Seo, B.-M.; Park, Y.J J; Hong, S.-D.; Roh, S.; Cho, J. J .; Park, W. H.; Min, B.-M., Electrospinning of collagen nanofibers: Effects on the behavior of normal human keratinocytes and early-stage wound healing. Biomaterials 2006, $27(8), 1452-1461$.

9. Ju, H. W.; Lee, O. J .; Lee, J . M.; Moon, B. M.; Park, H. J .; Park, Y. R.; Lee, M. C.; Kim, S. H.; Chao, J . R.; Ki, C. S.; Park, C. H., Wound healing effect of electrospun silk 
fibroin nanomatrix in burn-model. International Journal of Biological Macromolecules

$2016,85,29-39$.

10. Chen, J.-P.; Chang, G.-Y.; Chen, J.-K., Electrospun collagen/chitosan

nanofibrous membrane as wound dressing. Colloids and Surfaces A: Physicochemical

and Engineering Aspects 2008, 313-314, 183-188.

11. Ye, S.; J iang, L.; Wu, J .; Su, C.; Huang, C.; Liu, X.; Shao, W., Flexible

Amoxicillin-G rafted Bacterial C ellulose Sponges for Wound Dressing: In Vitro and in

Vivo Evaluation. ACS Applied Materials \& Interfaces 2018, 10 (6), 5862-5870.

12. Abdel-Mohsen, A. M.; J ancar, J .; Abdel-Rahman, R. M.; Vojtek, L.; Hyršl, P.;

Dušková, M.; Nejezchlebová, H., A novel in situ silver/hyaluronan bio-nanocomposite fabrics for wound and chronic ulcer dressing: In vitro and in vivo evaluations.

International Journal of Pharmaceutics 2017, 520 (1), 241-253. 
13. Miguel, S. P.; Figueira, D. R.; Simões, D.; Ribeiro, M. P.; Coutinho, P.; Ferreira, P.; Correia, I. J ., Electrospun polymeric nanofibres as wound dressings: A review. Colloids and Surfaces B: Biointerfaces 2018, 169, 60-71.

14. Wu, H.; Xiao, D.; Lu, J .; Jiao, C.; Li, S.; Lei, Y.; Liu, D.; Wang, J .; Zhang, Z.; Liu, Y.; Shen, G.; Li, S., Effect of high-pressure homogenization on microstructure and properties of pomelo peel flour film-forming dispersions and their resultant films. Food Hydrocolloids 2020, 102, 105628.

15. Adeli, H.; Khorasani, M. T.; Parvazinia, M., Wound dressing based on electrospun PVA/chitosan/starch nanofibrous mats: Fabrication, antibacterial and cytocompatibility evaluation and in vitro healing assay. International Journal of Biological Macromolecules 2019, 122, 238-254.

16. Khil, M. S.; Cha, D. I.; Kim, H. Y.; Kim, I. S.; Bhattarai, N., Electrospun nanofibrous polyurethane membrane as wound dressing. Journal of Biomedical Materials Research Part B-Applied Biomaterials 2003, 67B (2), 675-679. 
17. Fan, Z.; Liu, B.; Wang, J .; Zhang, S.; Lin, Q.; Gong, P.; Ma, L.; Yang, S., A Novel

Wound Dressing Based on Ag/G raphene Polymer Hydrogel: Effectively Kill Bacteria and

Accelerate Wound Healing. Advanced Functional Materials 2014, 24 (25), 3933-3943.

18. Kumbar, S. G.; Nukavarapu, S. P.; J ames, R.; Nair, L. S.; Laurencin, C. T.,

Electrospun poly(lactic acid-co-glycolic acid) scaffolds for skin tissue engineering.

Biomaterials 2008, 29 (30), 4100-4107.

19. Suwantong, O., Biomedical applications of electrospun polycaprolactone fiber

mats. Polymers for Advanced Technologies 2016, $27(10)$, 1264-1273.

20. Tra Thanh, N.; Ho Hieu, M.; Tran Minh Phuong, N.; Do Bui Thuan, T.; Nguyen

Thi Thu, H.; Thai, V. P.; Do Minh, T.; Nguyen Dai, H.; Vo, V. T.; Nguyen Thi, H.,

Optimization and characterization of electrospun polycaprolactone coated with gelatin-

silver nanoparticles for wound healing application. Materials Science and Engineering:

C2018, 91, 318-329. 
21. Aljghami, M. E.; Saboor, S.; Amini-Nik, S., Emerging Innovative Wound

Dressings. Annals of Biomedical Engineering 2019, 47(3), 659-675.

22. Furfaro, L. L.; Payne, M. S.; Chang, B. J ., Bacteriophage Therapy: Clinical Trials and Regulatory Hurdles. Frontiers in Cellular and Infection Microbiology 2018, 8, 376.

23. Ramalingam, R.; Dhand, C.; Leung, C. M.; Ong, S. T.; Annamalai, S. K.;

Kamruddin, M.; Verma, N. K.; Ramakrishna, S.; Lakshminarayanan, R.; Arunachalam,

K. D., Antimicrobial properties and biocompatibility of electrospun poly- $\varepsilon$-caprolactone

fibrous mats containing Gymnema sylvestre leaf extract. Materials Science and

Engineering: C 2019, 98, 503-514.

24. Zhang, W. W.; Ronca, S.; Mele, E., Electrospun Nanofibres Containing

Antimicrobial Plant Extracts. Nanomaterials 2017, $7(2), 42$.

25. Scaffaro, R.; Lopresti, F., P rocessing, structure, property relationships and release kinetics of electrospun PLA/Carvacrol membranes. European Polymer Journal

2018, 100, 165-171. 
26. Figueroa-Lopez, K. J .; Vicente, A. A.; Reis, M. A. M.; Torres-Giner, S.; Lagaron,

J. M., Antimicrobial and Antioxidant Performance of Various Essential Oils and Natural

Extracts and Their Incorporation into Biowaste Derived Poly(3-hydroxybutyrate-co-3-

hydroxyvalerate) Layers Made from Electrospun UItrathin Fibers. Nanomaterials 2019, 9

(2), 144.

27. Yildiz, Z. I.; Celebioglu, A.; Kilic, M. E.; Durgun, E.; Uyar, T., Fast-dissolving

carvacrol/cyclodextrin inclusion complex electrospun fibers with enhanced thermal

stability, water solubility, and antioxidant activity. Journal of Materials Science 2018, 53

(23), 15837-15849.

28. Scaffaro, R.; Lopresti, F.; D'Arrigo, M.; Marino, A.; Nostro, A., Efficacy of

poly(lactic acid)/carvacrol electrospun membranes against $\mathrm{Staphylococcus} \mathrm{aureus} \mathrm{and}$

Candida albicans in single and mixed cultures. Applied Microbiology and Biotechnology

2018, $102(9), 4171-4181$. 
29. Celebioglu, A.; Yildiz, Z. I.; Uyar, T., Thymol/cyclodextrin inclusion complex nanofibrous webs: Enhanced water solubility, high thermal stability and antioxidant property of thymol. Food Research Internationa/ 2018, 106, 280-290.

30. Gámez, E.; Mendoza, G.; Salido, S.; Arruebo, M.; Irusta, S., Antimicrobial Electrospun Polycaprolactone-Based Wound Dressings: An In Vitro Study About the Importance of the Direct Contact to Elicit Bactericidal Activity. Advances in Wound Care $2019,8,438-451$.

31. Yang, S. B.; Han, X. G.; J ia, Y. H.; Zhang, H. B.; Tang, T. T., Hydroxypropyltrimethyl Ammonium Chloride Chitosan Functionalized-P LGA Electrospun Fibrous Membranes as Antibacterial Wound Dressing: In Vitro and In Vivo Evaluation. Polymers 2017, 9 (12), 697.

32. Bui, H. T.; Chung, O. H.; Dela Cruz, J .; Park, J. S., Fabrication and characterization of electrospun curcumin-loaded polycaprolactone-polyethylene glycol nanofibers for enhanced wound healing. Macromolecular Research 2014, 22 (12), 1288-1296. 
33. Gamez, E.; Mendoza, G.; Salido, S.; Arruebo, M.; Irusta, S., Antimicrobial

Electrospun Polycaprolactone-Based Wound Dressings: An In Vitro Study About the Importance of the Direct Contact to Elicit Bactericidal Activity. Advances in Wound Care $2019,8(9), 438-451$.

34. Wang, X.; Ge, J .; Tredget, E. E.; Wu, Y., The mouse excisional wound splinting model, including applications for stem cell transplantation. Nature Protocols 2013, 8(2), 302-309.

35. Galiano, R. D.; Michaels, V., J oseph; Dobryansky, M.; Levine, J . P.; Gurtner, G. C., Quantitative and reproducible murine model of excisional wound healing. Wound Repair and Regeneration 2004, 12 (4), 485-492.

36. https://medicalguidelines.msf.org/viewport/CG/english/dressings-

18482377.html\#id-.DressingsvEnglish-Removalofanolddressing (accessed November 18).

37. https://www.ncbi.nlm.nih.gov/books/NBK53732/ (accessed November 18). 
38. Chen, Y.; Qiu, Y.; Chen, W.; Wei, Q., Electrospun thymol-loaded porous cellulose acetate fibers with potential biomedical applications. Materials Science and Engineering: C 2020, 109, 110536.

39. Del Nobile, M. A.; Conte, A.; Incoronato, A. L.; Panza, O., Antimicrobial efficacy and release kinetics of thymol from zein films. Journal of Food Engineering 2008, 89 (1), $57-63$.

40. Tampau, A.; González-Martínez, C.; Chiralt, A., Release kinetics and antimicrobial properties of carvacrol encapsulated in electrospun poly-( $\varepsilon$-caprolactone) nanofibres. Application in starch multilayer films. Food Hydrocolloids 2018, 79, 158-169.

41. Tampau, A.; González-Martinez, C.; Chiralt, A., Carvacrol encapsulation in starch or PCL based matrices by electrospinning. Journal of Food Engineering 2017, 214, 245256. 
42. Ramamoorthy, M.; Rajiv, S., In-vitro release of fragrant I-carvone from electrospun poly( $\square$-caprolactone)/wheat cellulose scaffold. Carbohydrate Polymers $2015,133,328-336$.

43. Bose, S.; Vu, A. A.; Emshadi, K.; Bandyopadhyay, A., Effects of polycaprolactone on alendronate drug release from $\mathrm{Mg}$-doped hydroxyapatite coating on titanium. Materials Science and Engineering: C2018, 88, 166-171.

44. Goonoo, N.; Bhaw-Luximon, A.; J hurry, D., Drug Loading and Release from Electrospun Biodegradable Nanofibers. Journal of Biomedical Nanotechnology 2014, 10 (9), 2173-2199.

45. Peppas, N. A.; Sahlin, J. J., A simple equation for the description of solute release. III. Coupling of diffusion and relaxation. International Journal of Pharmaceutics $1989,57(2), 169-172$. 
46. Trinca, R. B.; Westin, C. B.; da Silva, J . A. F.; Moraes, Â. M., Electrospun

multilayer chitosan scaffolds as potential wound dressings for skin lesions. European Polymer Journal 2017, 88, 161-170.

47. Mishra, R. K.; Mishra, P.; Verma, K.; Mondal, A.; Chaudhary, R. G .; Abolhasani, M. M.; Loganathan, S., Electrospinning production of nanofibrous membranes. Environmental Chemistry Letters 2019, $17(2), 767-800$.

48. Salehi, M.; Niyakan, M.; Ehterami, A.; Haghi-Daredeh, S.; Nazarnezhad, S.; Abbaszadeh-Goudarzi, G.; Vaez, A.; Hashemi, S. F.; Rezaei, N.; Mousavi, S. R., Porous electrospun poly( $\varepsilon$-caprolactone)/gelatin nanofibrous mat containing cinnamon for wound healing application: in vitro and in vivo study. Biomedical Engineering Letters 2020, $10(1), 149-161$.

49. Wang, B.; Zheng, H.; Chang, M.-W.; Ahmad, Z.; Li, J .-S., Hollow polycaprolactone composite fibers for controlled magnetic responsive antifungal drug release. Colloids and Surfaces B: Biointerfaces 2016, 145, 757-767. 
50. Quartinello, F.; Tallian, C.; Auer, J .; Schon, H.; Vielnascher, R.; Weinberger, S.;

Wieland, K.; Weihs, A. M.; Herrero-Rollett, A.; Lendl, B.; Teuschl, A. H.; Pellis, A.;

Guebit, G. M., S mart textiles in wound care: functionalization of cotton/PET blends with antimicrobial nanocapsules. Journal of Materials Chemistry B 2019, 7(42), 6592-6603.

51. García-Salinas, S.; Elizondo-Castillo, H.; Arruebo, M.; Mendoza, G .; Irusta, S., Evaluation of the Antimicrobial Activity and Cytotoxicity of Different Components of Natural O rigin Present in Essential Oils. Molecules (Basel, Switzerland) 2018, 23 (6), 1399.

52. Pérez-Recalde, M.; Ruiz Arias, I. E.; Hermida, É. B., Could essential oils enhance biopolymers performance for wound healing? A systematic review.

Phytomedicine 2018, 38, 57-65.

53. Karami, Z.; Rezaeian, I.; Zahedi, P.; Abdollahi, M., Preparation and performance evaluations of electrospun poly(epsilon-caprolactone), poly(lactic acid), and their hybrid (50/50) nanofibrous mats containing thymol as an herbal drug for effective wound healing. Journal of Applied Polymer Science 2013, 129 (2), 756-766. 
54. Riella, K. R.; Marinho, R. R.; Santos, J. S.; Pereira-Filho, R. N.; Cardoso, J. C.; Albuquerque-J unior, R. L.; Thomazzi, S. M., Anti-inflammatory and cicatrizing activities of thymol, a monoterpene of the essential oil from Lippia gracilis, in rodents. Journal of ethnopharmacology 2012, $143(2), 656-63$.

55. J iji, S.; Udhayakumar, S.; Rose, C.; Muralidharan, C.; Kadirvelu, K., Thymol enriched bacterial cellulose hydrogel as effective material for third degree burn wound repair. International Journal of Biological Macromolecules 2019, 122, 452-460.

56. Lin, Z.; Wu, T.; Wang, W.; Li, B.; Wang, M.; Chen, L.; Xia, H.; Zhang, T., Biofunctions of antimicrobial peptide-conjugated alginate/hyaluronic acid/collagen wound dressings promote wound healing of a mixed-bacteria-infected wound. International Journal of Biological Macromolecules 2019, 140, 330-342.

57. Sarhan, W. A.; Azzazy, H. M. E.; El-Sherbiny, I. M., Honey/Chitosan Nanofiber Wound Dressing Enriched with Allium sativum and Cleome droserifolia: Enhanced Antimicrobial and Wound Healing Activity. ACS Applied Materials \& Interfaces 2016, 8 (10), 6379-6390. 


\section{Graphical abstract}

(1)

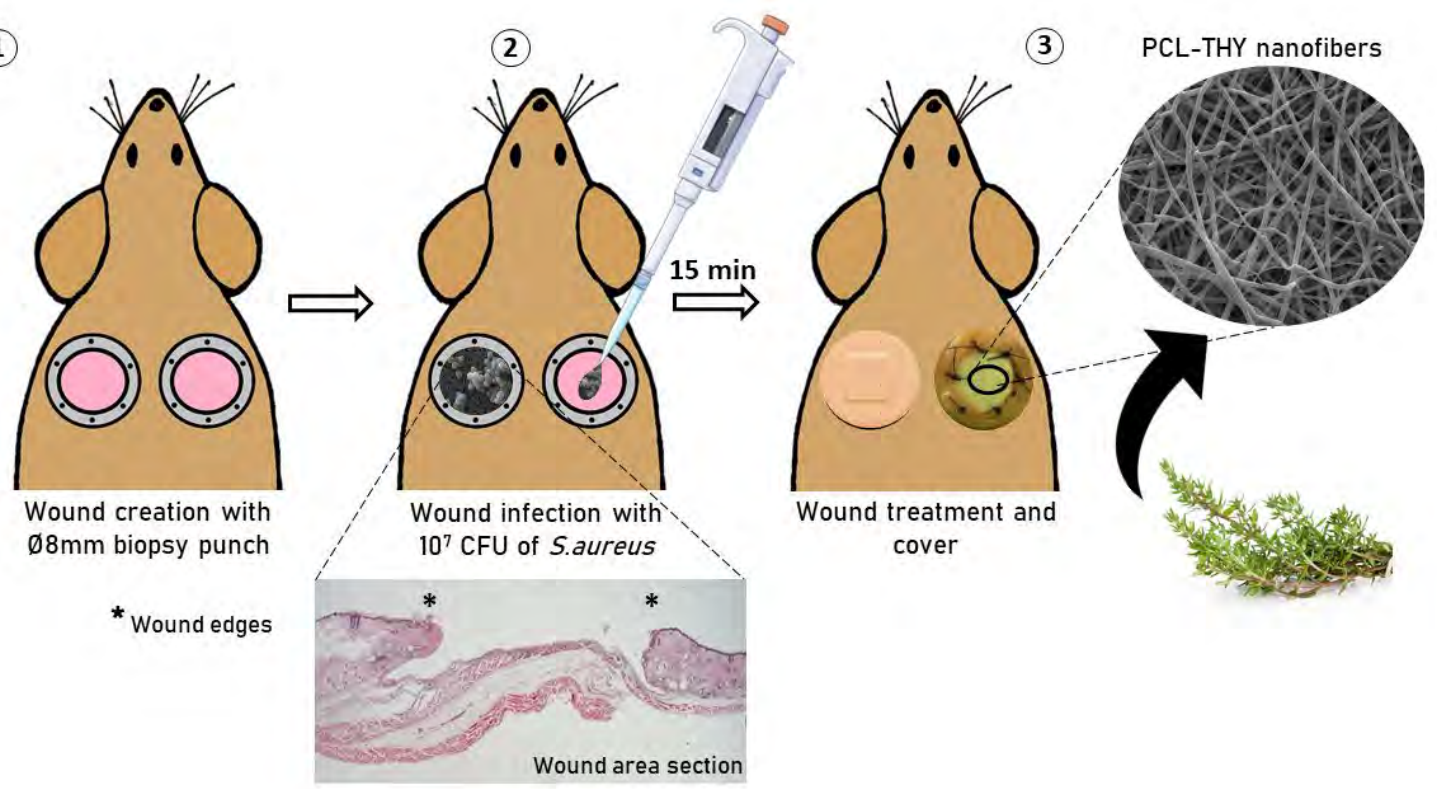

